\title{
Magnetic-reconnection exhausts in the sheath of magnetic clouds
}

\author{
Hengqiang Feng ${ }^{1,2}$ and Jiemin Wang ${ }^{1}$
}

\author{
${ }^{1}$ College of Physics and Electronic Information, Luoyang Normal University, 471022 Luoyang, PR China \\ e-mail: fenghq9921@163.com \\ 2 State Key Laboratory of Space Weather, Center for Space Science and Applied Research, Chinese Academy of Sciences, \\ 100190 Beijing, PR China
}

Received 22 August 2013 / Accepted 15 October 2013

\begin{abstract}
Context. Reconnection exhausts are a common phenomenon in the solar wind. Many exhausts were observed between an interplanetary coronal mass ejection (ICME) and the ambient solar wind, or between two ICMEs, or within the interior of a single ICME. Observed exhausts are almost exclusively associated with ICMEs with low (often much lower than 1) proton beta.

Aims. Often the sheath regions of ICMEs have a high level of plasma beta. Therefore we aim to find out whether the reconnection exhausts occur frequently in the sheath regions of ICMEs.

Methods. We examined the plasma and magnetic-field data in the sheath of the magnetic cloud (i.e., ICME) observed on 18-20 October 1995, and identified six reconnection exhausts.

Results. The six reconnection exhausts occured within regions of proton beta that was higher than unity. Five of them occurred on a high level ( $>2.2)$ of proton beta.

Conclusions. Low proton beta is no exclusive condition for magnetic reconnection. Reconnection may occur frequently in the sheath of ICMEs when magnetic fields from different source regions, that is, from diffident orientations, are pushed together.
\end{abstract}

Key words. solar-terrestrial relations - solar wind - Sun: magnetic fields

\section{Introduction}

Magnetic clouds (MCs) are an important subset of interplanetary coronal mass ejections (ICMEs), which are interplanetary manifestations of transient events with large amounts of material ejected from the solar atmosphere (e.g., Hundhausen 1987; Kahler 1987). An MC was originally defined empirically in terms of in situ spacecraft measurements of magnetic fields and plasma in the interplanetary medium at $\sim 1 \mathrm{AU}$; that is, it has the following necessary properties: (1) a higher magnetic-field magnitude than the ambient field; (2) a smooth change in field direction as observed by a spacecraft passing through the cloud; and (3) a lower proton temperature than the ambient proton temperature (Burlaga et al. 1981, 1990; Burlaga 1995). MCs are typically observed in association with bidirectional ion and electron beams (e.g., Bame et al. 1981; Gosling et al. 1987; Marsden et al. 1987), helium enhancements (Zwickl et al. 1983), and decreases in the energetic particle intensity (Cane et al. 1997). MCs have received considerable attention because the magnetic configuration is amenable to simple flux-rope modeling (e.g., Goldstein 1983; Burlaga 1988, 1991; Lepping et al. 1990; Farrugia et al. 1995), and they are responsible for some major geomagnetic storms (e.g., Webb et al. 2000). When an MC propagates outward from the Sun, the faster-moving MC will compress and deflect the upstream flow. If the relative speed of the two plasma regimes is higher than the fast-mode magnetohydrodynamic (MHD) wave speed, a shock will form ahead of the MC. The region of compressed solar wind bounded by the leading shock front and the leading edge of the $\mathrm{MC}$-front is referred to as the sheath region. The sheath regions often have high plasma beta, density, proton temperature, and magnetic-field magnitude (e.g., Guo et al. 2010, 2011). Within a sheath, the dynamic pressure is typically high and fluctuates more than within MCs, and the magnetic-field direction can change many times. The sheath durations range from 7.7 to $19.1 \mathrm{~h}$ with an average value of $14.8 \mathrm{~h}$ (Feng et al. 2010).

Magnetic reconnection is an important and efficient process that converts magnetic energy into bulk-flow energy (particle energy). This process generally favors oppositely directed magnetic fields at thin current sheets and plays a central role in many interpretations and models of space, solar, astrophysical, and laboratory plasma phenomena (e.g., Parker 1983; Priest \& Forbes 2000; Priest et al. 2005). Recently, observational studies have focused on some of these macroscopic properties of magnetic reconnection. Gosling et al. (2005a) first developed magnetic-reconnection exhaust criteria based on the Petschek reconnection model (Petschek 1964). The exhausts are roughly Alfvénic plasma-jet flows confined to magnetic-field reorientation regions, which typically take the form of bifurcated current sheets. The direct evidence to identify reconnection exhausts in the solar wind is a pair of correlated and anti-correlated variations in $\boldsymbol{B}$ and $\boldsymbol{V}$ at the edges of the exhaust. The reconnection exhausts reported by Gosling et al. (2005a) have the following characteristics: within the exhaust the plasma beta, the proton temperature, and the density are all higher than outside if the magnetic-field strength is weaker than that of the surrounding solar wind; the field shear across these exhausts range is large (often $>98^{\circ}$ ); and the surrounding solar wind has low proton beta $(<0.15)$. Since ICMEs (i.e., MCs) have low (often $\ll 1)$ proton beta, many reconnection events have been found to be associated with ICMEs (e.g., Gosling et al. 2007; Gosling \& Szabo 2008; Lugaz et al. 2005), and a number of small field-shear angle exhausts occurred within the interiors of ICMEs (Gosling $\&$ Szabo 2008), even certain exhausts within the ICME were 


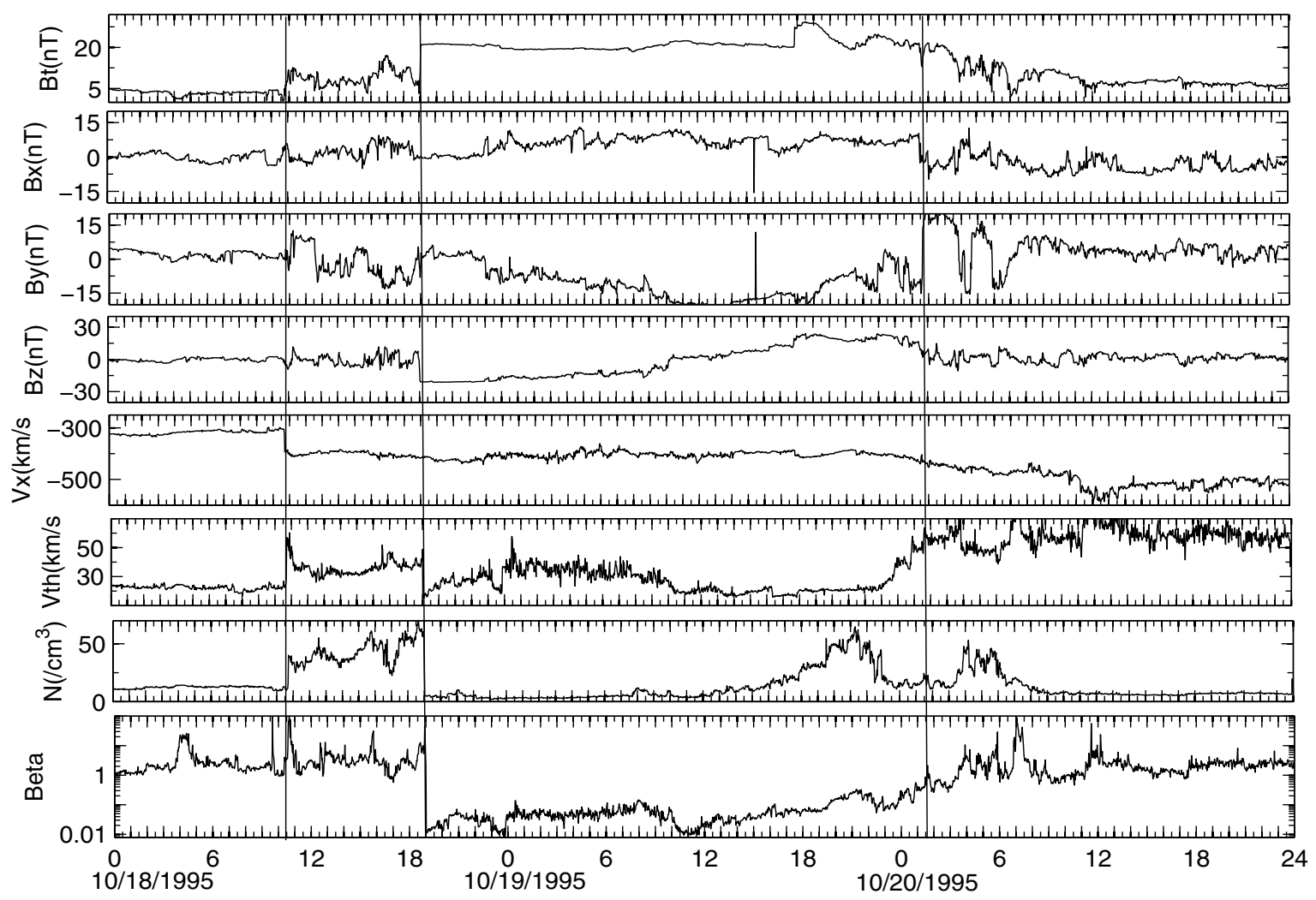

Fig. 1. Interplanetary magnetic field and plasma data measured by the WIND spacecraft in the GSE coordinate system during the passage of the MC on 18-20 October 1995.

associated with current sheets having extremely small $\left(4^{\circ}\right)$ fieldshear angles (Gosling \& Phan 2013). Accordingly, a large fieldshear angle is not the only condition for magnetic reconnection. Similar, it is interesting to ask whether magnetic reconnections can occur in the solar wind with high proton beta $(>2)$ ? As mentioned above, the sheath regions of MCs often have high proton beta. In addition, signatures of magnetic reconnection have previously been found in the fronts of MCs (e.g., Farrugia et al. 2001; Ruffenach et al. 2012). Can magnetic reconnections occur frequently in the sheath regions of MCs? We here report six reconnection exhausts in the sheath of MC observed by WIND on 18-20 October 1995, all six of which were observed in plasma characterized by high proton beta.

\section{Observations}

We used 3-s-averaged plasma and magnetic-field data measured by WIND, the magnetic-field data were obtained from Magnetic Field Investigation (MFI) magnetometer and the proton data are obtained from the 3-Dimension Plasma (3DP) analyzer. A description of the instruments onboard WIND was given by Lepping et al.(1995) and Lin et al. (1995). Figure 1 shows the interplanetary magnetic field and plasma, which were measured in the GSE during the passage of the MC on 18-20 October 1995. From top to bottom the panels show the magnitude of the total magnetic field $\left(B_{\mathrm{t}}\right)$, the $x, y, z$ components of the magnetic field $\left(B_{x}, B_{y}, B_{z}\right)$, the solar wind velocity along the Sun-Earth direction $\left(V_{x}\right)$, the proton thermal speed $\left(V_{\text {th }}\right)$ and density $(N)$, and the proton beta, respectively. The MC has been discussed by many authors (e.g., Lepping et al. 1997; Wu et al. 2000; Wei et al. 2003; Feng et al. 2006). Clearly there is a preceding shock produced by the MC at 10:56 UT on 18 October 1995. There is a distinct directional discontinuity (DD) at 18:58 UT, which was identified as the front boundary of the MC by many authors (e.g., Wu et al. 2000; Wei et al., 2003; Feng et al. 2006). The leading shock and the front boundary are denoted by two vertical solid lines in Fig. 1, this time interval defines the MC sheath. For the MC, different authors gave different rear boundaries due to the ambiguities properties, and we identified the rear boundary at 01:33 UT on 18 October 1995 (Feng et al. 2006). However, a different rear boundary was identified by Dasso et al. (2006) at 17:37 UT on 19 October 199. But the difference in rear boundaries dose not affect the identification of the MC sheath. The MC was characterized by smooth magnetic-field rotations, low proton number density, and low proton beta. In contrast,the magnetic-field direction in the MC sheath was fluctuating; the proton beta within the sheath varied from 0.06 to 44 with a mean value of 3.84, which is much higher than the average proton beta (0.076) within the MC.

We identified six reconnection exhausts within the sheath of the MC on 18-20 October 1995, two of which occurred immediately at the beginning and two at the end of the sheath. Figure 2 provides expanded views of the magnetic field and velocity curves of the magnetic-reconnection exhaust. The reconnection exhaust occurred during 11:14:34-11:14:58 UT. As can be seen from Fig. 2, the changes in $\boldsymbol{V}$ and $\boldsymbol{B}$ are correlated at its leading edge and are anti-correlated at its trailing edge. In addition, the exhaust is characterized by increases in proton density, temperature, and proton beta within the exhaust, the magneticfield strength is weaker than that of the surrounding solar wind (not shown in Fig. 2). The change of the observed field orientation in this exhaust is strong $\left(102^{\circ}\right)$. These features are consistent with the previously reported reconnection exhausts. However, the ambient solar wind has a high level of proton beta. The proton beta is 1.06 at its leading edge and 2.54 at its trailing edge. This indicates that magnetic reconnection can occur at the 
H. Feng and J. Wang: Magnetic-reconnection exhausts in the sheath of magnetic clouds

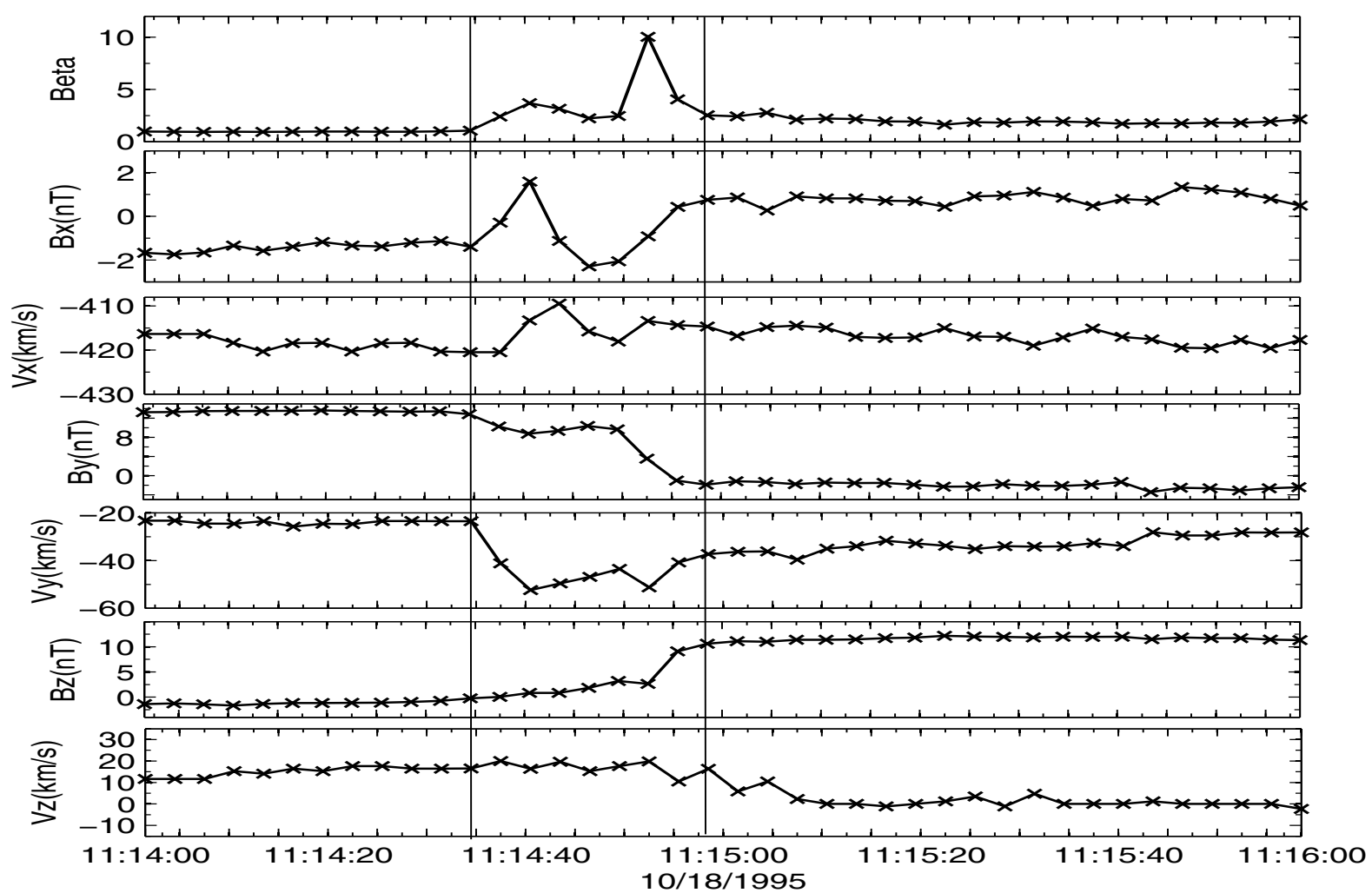

Fig. 2. Interplanetary magnetic field, speed data and proton beta measured by the WIND spacecraft during the 11:14:34-11:14:58 UT magnetic reconnection exhaust passage.

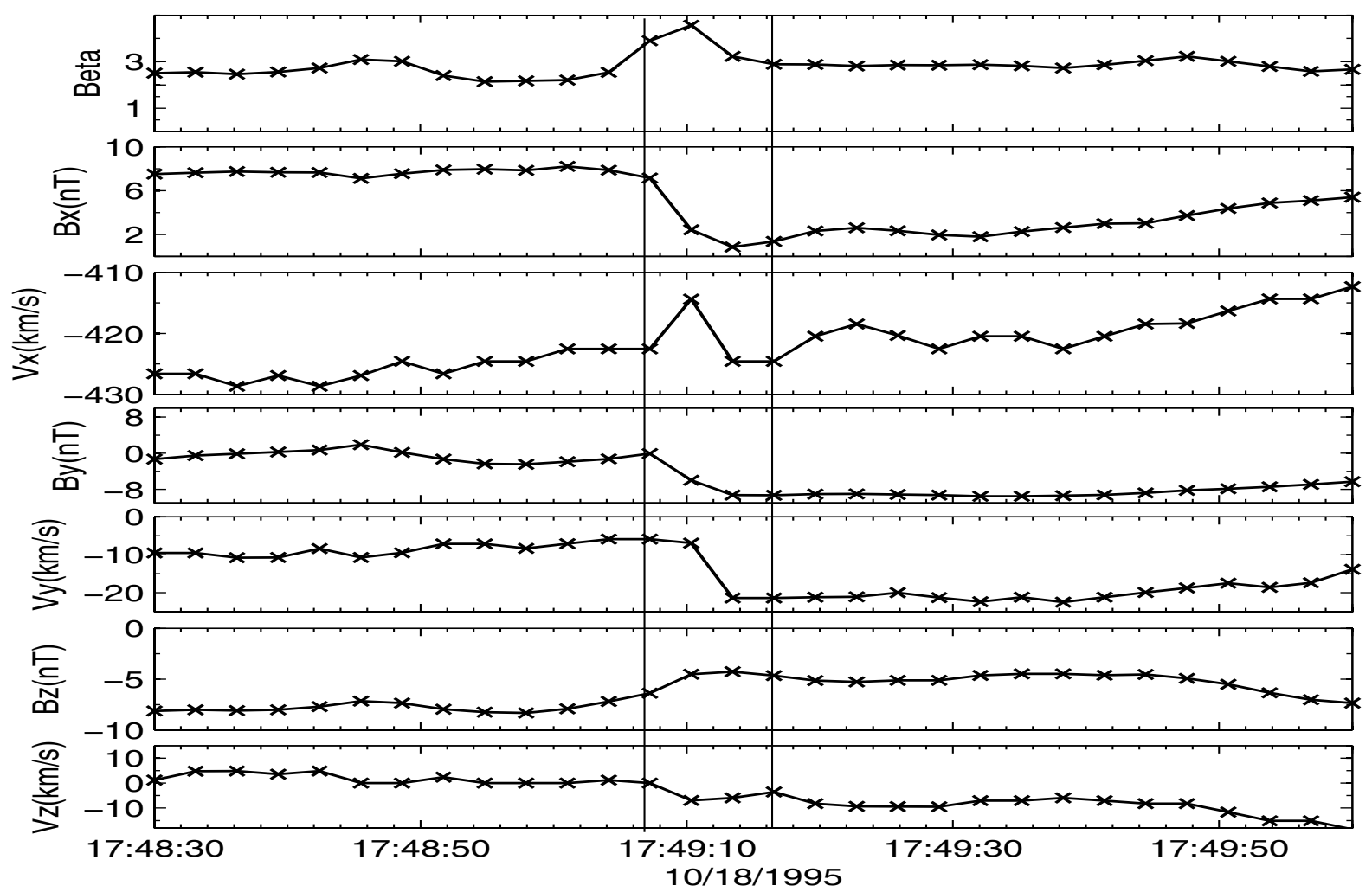

Fig. 3. Interplanetary magnetic field, speed data, and proton beta measured by the WIND spacecraft during the 17:49:07-17:49:16 UT magneticreconnection exhaust passage.

interface between two different plasma flows characterized by high $(>1)$ proton beta.

Figure 3 shows the exhaust event within 17:49:0717:49:16 UT. The changes in $\boldsymbol{V}$ and $\boldsymbol{B}$ were anti-correlated with one another at the leading edge and were correlated at the trailing edge. During the exhaust, the proton density, temperature, and proton beta are all enhanced, whereas the field strength is diminished (not shown). All the above properties are characteristic of 
Table 1. Observational characteristics of reconnection exhausts.

\begin{tabular}{lccccc}
\hline \hline $\mathrm{N}^{\circ}{ }^{a}$ & Start $^{b}$ & End $^{c}$ & Angle $^{d}$ & $\beta_{1}{ }^{e}$ & $\beta_{2}{ }^{f}$ \\
\hline 01 & $11: 02: 07$ & $11: 02: 28$ & 36 & 3.92 & 3.56 \\
02 & $11: 14: 34$ & $11: 14: 58$ & 102 & 1.06 & 2.54 \\
03 & $14: 06: 16$ & $14: 06: 22$ & 36 & 2.65 & 2.28 \\
04 & $14: 16: 34$ & $14: 17: 07$ & 9 & 2.29 & 2.49 \\
05 & $17: 45: 01$ & $17: 45: 22$ & 85 & 3.74 & 2.61 \\
06 & $17: 49: 07$ & $17: 49: 16$ & 67 & 3.90 & 2.89 \\
\hline
\end{tabular}

Notes. ${ }^{(a)}$ The code number of the reconnection exhaust. ${ }^{(b)}$ The beginning of the reconnection exhaust (UT). ${ }^{(c)}$ The end of the reconnection exhaust (UT). ${ }^{(d)}$ The magnetic-field shear angle across the reconnection exhaust (degree). ${ }^{(e)}$ The proton beta at the leading edge of the reconnection exhaust. ${ }^{(f)}$ The proton beta at the trailing edge of the reconnection exhaust.

reconnection exhausts. In addition, the exhaust occurred within a solar wind in quite high $(>2.5)$ proton beta. The proton beta at the leading edge is 3.90 , that at the trailing edge is 2.89 . Table 1 lists the six identified magnetic-reconnection exhausts. Table 1 indicates that the timescale of these six exhausts ranges from 6 to $32 \mathrm{~s}$. The observed magnetic-field shears across the exhausts range from $9^{\circ}$ to $102^{\circ}$, and the external proton betas range from 1.06 to 3.92 with a mean value of 2.83 .

\section{Discussion}

Recently, many reconnection exhaust observations in the solar wind were reported (e.g., Gosling et al. 2005a,b, 2006a,b; Phan et al. 2006). A large number of these reconnection exhausts events have been found to be associated with ICMEs. These reconnection exhausts can be classified into three types based on the occurrence locations: (1) between ICMEs and the ambient solar wind; (2) between ICME and ICME; and (3) within the interior of ICMEs (Xu et al. 2011). In addition, Gosling et al. (2007) have pointed out that these exhausts were observed almost exclusively in either the low-speed wind or in association with ICMEs in plasma with predominantly low (often $\ll 1$ ) proton beta. However, the sheath regions of ICMEs (MCs) often have a high level of proton beta. Could the reconnection exhausts also exclusively occur in the sheath regions of ICMEs? We examined the plasma and magnetic-field data of the sheath of the MC observed on 18-20 October 1995, and identified six reconnection exhausts. Except for the reconnection exhaust that occurred at 11:14:34-11:14:58 UT, the reconnection events occurred in the ambient solar wind that had quite a high $(>2.2)$ proton beta level. Therefore, a low level of proton beta is not the only condition for magnetic reconnection. In addition, solar wind regions on opposite sides of the reconnection exhausts typically had small, but distinctly different, plasma and field characteristics, (e.g., Gosling et al. 2006a), that is, magnetic reconnection occurs at the interface between different streams. The sheath regions often have highly fluctuating fields, density, and dynamic pressure; it may contain many different interacting streams. Ruffenach et al. (2012) also found evidences of exhaust at the front boundary of $\mathrm{MC}$, which means that magnetic reconnections may occur frequently in the sheath of ICME.

Acknowledgements. This work is supported by National Nature Science Foundation of China (NSFC) under grant Nos. 41074124, 41274180, and 41231068. This work is also sponsored by the Program for Science and Technology Innovation Research Team in University of Henan Province Grant
No.13IRTSTHN020, and by a Project Supported by the Specialized Research Fund for State Key Laboratories. This work is supported in part by Program for Science \& Technology of Henan under grant Nos. 122300410331. The authors thank NASA/GSFC for the use of data from Wind.

\section{References}

Bame, S. J., Asbridge, J. R., Feldman, W. C., Gosling, J. T., \& Zwickl, R. D. 1981, Geophys. Res. Lett., 8, 173

Burlaga, L. F. 1995, in Interplanetary magnetohydrodynamics, International Series in Astronomy and Astrophysics (Oxford University Press), 3

Burlaga, L., Sittler, E., Mariani, F., \& Schwenn, R. 1981, J. Geophys. Res., 86, 6673

Burlaga, L. F., Lepping, R. P., \& Jones, J. A. 1990 (Washington DC: American Geophysical Union), in Physics of magnetic flux ropes (A92-31201 12-75), 58,373

Burlaga, L. F., Hewish, A., \& Behannon, K. W. 1991, J. Geophys. Res., 96, 21213

Cane, H. V., Richardson, I. G., \& Wibberenz, G. 1997, J. Geophys. Res., 102, 7075

Dasso, S., Mandrini, C. H., Démoulin, P., \& Luoni, M. L. 2006, A\&A, 455, 349

Farrugia, C. J., Osherovich, V. A., \& Burlaga, L. F. 1995, J. Geophys. Res., 100, 12293

Farrugia, C. J., Vasquez, B., Richardson, I. G., et al. 2001, Adv. Space Res., 28, 759

Feng, H. Q., Wu, D. J., Chao, J. K., Lee, L. C., \& Lyu, L. H. 2010, J. Geophys. Res. (Space Physics), 115, A04107

Feng, H. Q., Wu, D. J., \& Chao, J. K. 2006, J. Geophys. Res. (Space Physics), $111, \mathrm{~A} 07 \mathrm{~S} 90$

Goldstein, H. 1983, NASA Conf. Publ., 228, 731

Gosling, J. T., \& Phan, T. D. 2013, ApJ, 763, L39

Gosling, J. T., \& Szabo, A. 2008, J. Geophys. Res. (Space Physics), 113, A10103

Gosling, J. T., Baker, D. N., Bame, S. J., et al. 1987, J. Geophys. Res., 92, 8519

Gosling, J. T., Skoug, R. M., McComas, D. J., \& Smith, C. W. 2005a, J. Geophys. Res. (Space Physics), 110, A01107

Gosling, J. T., Skoug, R. M., McComas, D. J., \& Smith, C. W. 2005b, Geophys. Res. Lett., 32, 5105

Gosling, J. T., Eriksson, S., Skoug, R. M., McComas, D. J., \& Forsyth, R. J. 2006a, ApJ, 644, 613

Gosling, J. T., Eriksson, S., \& Schwenn, R. 2006b, J. Geophys. Res., 111, A10102

Gosling, J. T., Eriksson, S., McComas, D. J., Phan, T. D., \& Skoug, R. M. 2007, J. Geophys. Res. (Space Physics), 112, A08106

Guo, J., Feng, X., Zhang, J., Zuo, P., \& Xiang, C. 2010, J. Geophys. Res. (Space Physics), 115, A09107

Guo, J., Feng, X., Emery, B. A., et al. 2011, J. Geophys. Res. (Space Physics), 116, A05106

Hundhausen, A. J. 1987, Sixth International Solar Wind Conference, 2, 181

Kahler, S. 1987, Sixth International Solar Wind Conference, 215

Lepping, R. P., Burlaga, L. F., \& Jones, J. A. 1990, J. Geophys. Res., 95, 11957

Lepping, R. P., Acũna, M. H., Burlaga, L. F., et al. 1995, Space Sci. Rev., 71, 207

Lepping, R. P., Burlaga, L. F., Szabo, A., et al. 1997, J. Geophys. Res., 102, 14049

Lin, R. P., Anderson, K. A., Ashford, S., et al. 1995, Space Sci. Rev., 71, 125

Lugaz, N., Manchester, W. B., IV, \& Gombosi, T. I. 2005, ApJ, 634, 651

Parker, E. N. 1983, ApJ, 264, 635

Petschek, H. E. 1964, NASA Spec. Publ., 50, 425

Marsden, R. G., Sanderson, T. R., Tranquille, C., Wenzel, K.-P., \& Smith, E. J. 1987, J. Geophys. Res., 92, 11009

Phan, T. D., Gosling, J. T., Davis, M. S., et al. 2006, Nature, 439, 175

Priest, E., \& Forbes, T. 2000, Magnetic reconnection: MHD theory and applications (New York: Cambridge University Press)

Priest, E. R., Longcope, D. W., \& Heyvaerts, J. 2005, ApJ, 624, 1057

Ruffenach, A., Lavraud, B., Owens, M. J., et al. 2012, J. Geophys. Res. (Space Physics), 117, A09101

Webb, D. F., Cliver, E. W., Crooker, N. U., Cry, O. C. S., \& Thompson, B. J. 2000, J. Geophys. Res., 105, A07491

Wei, F., Liu, R., Fan, Q., \& Feng, X. 2003, J. Geophys. Res. (Space Physics), 108, 1263

Wu, D. J., Chao, J. K., \& Lepping, R. P. 2000, J. Geophys. Res., 105, A12627

Xu, X., Wei, F., \& Feng, X. 2011, J. Geophys. Res. (Space Physics), 116, A05105

Zwickl, R. D., Asbridge, J. R., Bame, S. J., et al. 1983, NASA Conf. Publ., 228, 711 